\title{
2077. Noise reduction in centrifugal pump as turbine: influence of leaning blade or tongue
}

\author{
Cui Dai ${ }^{1}$, Liang Dong ${ }^{2}$, Bin $\mathrm{Xia}^{3}$, Fan Yu Kong ${ }^{4}$ \\ ${ }^{1}$ School of Energy and Power Engineering, Jiangsu University, Zhenjiang 212013, P. R. China \\ $2,3,{ }^{4}$ Research Center of Fluid Machinery Engineering and Technology, Jiangsu University, \\ Zhenjiang, 212013, P. R. China \\ ${ }^{2}$ Corresponding author

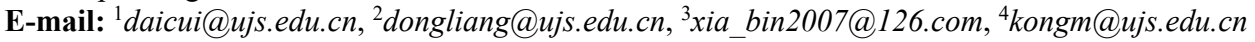

Received 8 January 2016; received in revised form 22 April 2016; accepted 24 May 2016

DOI http://dx.doi.org/10.21595/jve.2016.16811

\begin{abstract}
To reduce the interior/exterior flow-induced noise in centrifugal pump as turbine (PAT), based on the relation between in-phase hydrodynamic action and radiated noise, the angle formula in ideal condition by individually leaning blade was proposed. Considering the joint leaning effect, the formulas associated with parallel- and counter-leaning blade and tongue were presented. The corresponding active noise reduction approaches were put forward without losing hydraulic performance. For noise simulation, the numerical method for interior noise and finite element model for casing structure were checked, with good agreements achieved. The boundary element method (BEM) and finite element method (FEM) were used to investigate the interior/exterior noise characteristics of PATs by varying the shapes of blade or tongue. Researches show that the interior noise due to casing source can reflect joint action of multiple sources. It is feasible to predict the interior noise induced only by casing source through BEM. The leaned blade can keep PAT's original performance, the leaned tongue can significantly improve the efficiency overall operating range with an increase from $0.67 \%$ to $1.81 \%$, and the combined counter-leaning blade and tongue can increase the efficiency in larger flow rates. They can all reduce sound pressure level (SPL) at fundamental frequency and total sound energy, and the noise reduction effect under joint action is best.
\end{abstract}

Keywords: pump as turbine, leaning blade or tongue, boundary element method, finite element method, flow-induced noise.

\section{Nomenclature}

$\begin{array}{ll}b_{2} & \text { Impeller outlet width, } \mathrm{mm} \\ b_{3} & \text { Volute outlet width, } \mathrm{mm} \\ D_{1} & \text { Impeller inlet diameter, } \mathrm{mm} \\ D_{2} & \text { Impeller outlet diameter, } \mathrm{mm} \\ D_{4} & \text { Volute outlet diameter, } \mathrm{mm} \\ E & \text { Elastic module, } \mathrm{GPa} \\ f & \text { Frequency, } \mathrm{Hz} \\ H & \text { Head, } \mathrm{m} \\ n & \text { Rotating speed, } \mathrm{rpm} \\ P & \text { Power, } \mathrm{kw} \\ Q & \text { Flow rate, } \mathrm{m}^{3} / \mathrm{h} \\ Z & \text { Acoustic impedance, } \mathrm{kg} /\left(\mathrm{m}^{2} \cdot \mathrm{s}\right) \\ Z & \text { Blade number } \\ \beta & \text { Blade angle, }{ }^{\circ} \\ \gamma_{1 t} & \text { The circumferential leaned angle of blade in ideal state, }{ }^{\circ} \\ \gamma_{2 t} & \text { The circumferential leaned angle of tongue in ideal state, } \\ \eta & \text { Efficiency, } \% \\ \mu & \text { Poisson ratio } \\ \rho & \text { Density, } \mathrm{kg} / \mathrm{m}^{3}\end{array}$


Subscripts 1 Variable at low pressure end

Subscripts 2 Variable at high pressure end

\section{Introduction}

As an ideal method of energy saving technology by recovery of residual pressure, pump as turbine (PAT) has been widely used in many engineering fields, such as petroleum and chemical industry, small and micro-hydro plants and so on [1]. By PAT, the high pressure energy of liquid can be transformed to rotational mechanical energy of shaft, and then used to drive other energy-consuming equipment. The PAT with high power can further improve the utilization ratio of recovery system. However, the corresponding noise has become one of the most important issues that cause negative effect on reliability.

Currently, the noise reduction technology is becoming an international research focus. One of the most fundamental and effective methods to control noise is decreasing the noise source intensity radiated from solid surface. There are mainly two ways, that are active and passive noise control [2]. Active noise control is realized primarily by improving the shapes or materials of impeller, blade and pipeline. While, passive noise control focuses on minimizing the noise level spread to outside. Generally, it increases the energy consumption due to added isolation measures. With respect to active noise control, some earlier methods were based on the modifications of tongue geometry in order to minimize the pressure fluctuations in that zone. Tourret et al. [3] found the worst pressure fluctuation in a volute-type centrifugal pump was located at the tongue, and there were some certain relations between the pressure fluctuation and radiated noise on structural surface. Then, Dong et al. [4] studied the flow structure and resulting noise by modifying the tongue geometry based on Particle Image Velocimetry (PIV), pressure, and noise measurements. He pointed out that, the contribution to noise from jet/wake phenomenon was insignificant by increasing the gap between tongue and impeller. The major contributor to noise was tongue oscillations relating to its stiffness. Attempts can be made to reduce noise by increasing the gap, adding the tongue's stiffness, and attaching damping pieces at the tongue. After determining the importance of tongue shape on flow-induced noise, Langthjem et al. $[5,6]$ studied the relationship between the geometry of centrifugal pump and noise sources in it by establishing a two-dimensional mathematical model. As for the core impeller and blade in centrifugal pump, many researches have focused on the impact of the shape, size and corresponding unsteady hydrodynamic force on flow-induced noise. Neise [7] found that the blade can be slotted to reduce noise. In [4], an attempt was also made to reduce the outflow nouniformity from impeller by inserting short vanes between blades. From all the studies above, the effects of the geometric parameters of impeller and volute in centrifugal pump were usually studied to reduce flow-induced noise, and few literatures have been presented on the noise generation mechanism and control method for PAT.

Due to backward-curved blades only suitable for pump, a pump is not ideally designed to operate as a turbine. Unlike conventional turbine with guide vane system, when a pump works as turbine, the inlet blade angle is fixed overall operating range. A vortex region will be formed caused by inlet attack angle. And the pressure difference between volute inlet and impeller outlet increases with increasing flow rate, causing the leakage amount to increase [8]. So compared with centrifugal pump, the lower efficiency and higher flow-induced noise for PAT are usually obtained caused by the poor design of flow passage and impeller-volute matching. For PAT, considerable researches have dealt with hydraulic performance prediction used for type-selection $[9,10]$ and performance improvement with focusing on the impact of geometry parameters $[11,12]$. Only pressure fluctuation characteristics within flow passages in PAT were researched [13]. Dai et al. [14, 15] studied the radial exciting force characteristics inducing noise and optimized the hydraulic and noise property of PAT based on response surface method after analyzing the effect of six factors (inlet angle, outlet angle, outlet width, wrap angle, inlet diameter, and blade number) on interior noise. Dong et al. [16] studied the impact of blade outlet angle on 
interior/exterior noise of PAT.

In the work reported here, a theoretical analysis of noise reduction by leaning blade or tongue has been performed. And then, experimental studies on hydraulic performance, interior noise and structure modal of PAT were carried out to validate the accuracy of numerical method. Eventually, some different blade and tongue geometric configurations have been studied in order to evaluate the effects of leaned blade and leaned tongue and their coupling effect on hydraulic performance and noise of PAT. The fundamental object of the present work is to provide computing methods of interior and exterior noise verified by experiments. The second object is to confirm the feasibility of improvement of hydraulic and noise performance by leaning blade or tongue without decreasing hydraulic performance overall operating range. And the further object is to understand whether the effect of noise reduction by counter-leaning blade and tongue is better.

\section{Theory of active noise reduction by leaning blade or tongue}

The blade outlet gradually staggered from shroud to hub in circumferential direction is defined as leaned blade. When using it, each blade in PAT no longer sweeps across the tongue at one time, but gradually over a period of time. A certain phase difference of fluid from shroud to hub is produced due to the unsteady interaction between them. Then, the in-phase pulsating hydrodynamic area is decreased, and finally the radiated noise is reduced [2].

When adopting leaned blade, the rear and front end of trailing edge on one blade will overlap with the front and rear end of adjacent blade, respectively. Three overlapping states will be presented. As adjacent blade just overlaps each other (as shown in Fig. 1), the area sweeping across the tongue remains the same. And sustained equal pulsation level can be produced, which further reduces in-phase hydrodynamic action. At this time, the adjacent blades are completely overlapped, which means the rear and front end of trailing edge is always in perfect overlapping state with the front and rear end of adjacent blade (i.e., $A_{1}=A_{2}$ ). The following formula exists:

$$
\frac{b_{2}}{\tan \left(\gamma_{1 t}\right)} z=\pi D_{2} \text {. }
$$

The ideal circumferential leaned angle of blade can be deduced as:

$$
\gamma_{1 \mathrm{t}}=\arctan \left(\frac{z b_{2}}{\pi D_{2}}\right)
$$

where, $D_{2}$ is the impeller outlet diameter, $b_{2}$ is the impeller outlet width, $z$ is blade number, $\gamma_{1 t}$ is the circumferential leaned angle of blade in ideal state. Smaller $\gamma_{1 t}$ will improve the noise performance and lower the hydraulic performance. As can be seen from Eq. (2), the ideal leaned angle of blade is inversely proportional to outlet diameter, but proportional to outlet width and blade number.

\section{Rotation direction}

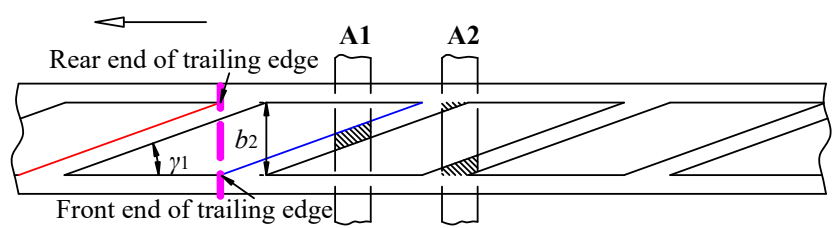

Fig. 1. Leaned blade in overlapping state

For low specific speed centrifugal pump in turbine mode, the ideal leaned angle of blade is smaller due to low ratio of outlet width to diameter $\left(b_{2} / D_{2}\right)$. Fig. 2 shows the relationship between the ideal leaned angle and geometric parameters inferred by Eq. (2). In which, $D_{2}$ ranges from 
$215 \mathrm{~mm}$ to $255 \mathrm{~mm}, b_{2}$ ranges from $11 \mathrm{~mm}$ to $15 \mathrm{~mm}$, and $z$ ranges from 5 to 12 . And, these parameters are normalized. It can be seen, within certain geometric parameters, the ideal leaned angle is relatively small, with a maximum angle of $14.95^{\circ}$. In the range of $D_{2}^{*}=[0,1]$, $b_{2}^{*}=[0,0.625]$ and $z^{*}=[0.5,1]$, the ideal leaned angle is larger. Under the combination of $D_{2}=255 \mathrm{~mm}, b_{2}=14 \mathrm{~mm}$ and $z=12, \gamma_{1 t}=11.84^{\circ}$ can completely meet the overlapping requirement.

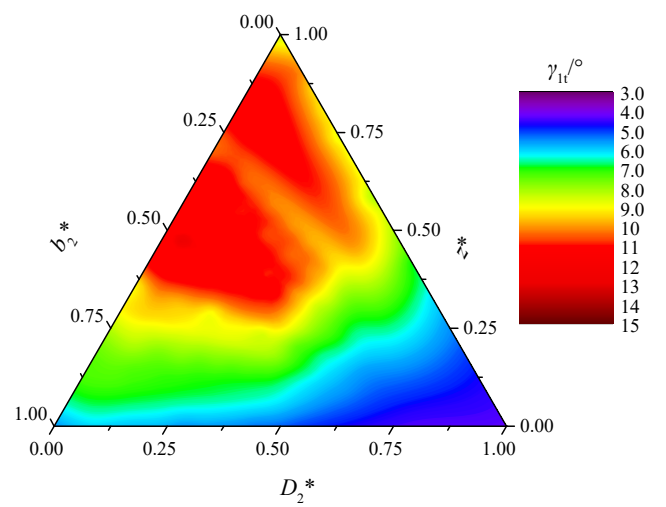

Fig. 2. Relation between ideal leaned angle of blade and geometric parameters

The hydraulic performance of PAT will be seriously deteriorated if $\gamma_{1 t}$ is too small. So, maybe the blade and tongue can pay their leaning share. That is, apart from leaning blade, the tongue of volute is also changed from common flat to leaned type. Different leaning ways of blade and tongue are shown in Fig. 3.

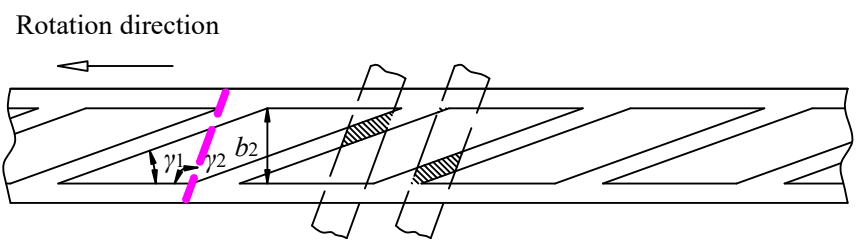

a) Parallel-leaning

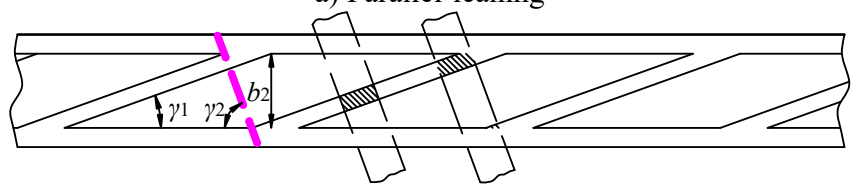

b) Counter-leaning

Fig. 3. Different leaning ways of blade and tongue

For parallel-leaned blade and tongue in ideal state, the following formula exists:

$\cot \gamma_{1 t}-\cot \gamma_{2 t}=\frac{\pi D_{2}}{z b_{2}}$

For counter-leaned blade and tongue in ideal state, the following formula exists:

$\cot \gamma_{1 t}+\cot \gamma_{2 t}=\frac{\pi D_{2}}{z b_{2}}$

where, $\gamma_{2 t}$ denotes the circumferential leaned angle of tongue in ideal state. Similarly, smaller $\gamma_{2 t}$ will improve the noise performance and lower the hydraulic performance. From Eqs. (1), (3) and 
(4), compared to other PATs with counter-leaned blade and tongue and flat tongue, the ideal leaned angle of blade under parallel leaning state increases, which decreases the noise performance. For counter-leaning state, under the parameter combination of $D_{2}=255 \mathrm{~mm}, b_{2}=$ $14 \mathrm{~mm}$ and $z=12, \gamma_{1 t}=11.84^{\circ}, \gamma_{2 t}=12.5^{\circ}$ can meet complete overlapping requirement. But due to the processing limits, the ideal leaned angle of tongue cannot be made too small.

\section{Numerical calculation of interior/exterior noise in PAT}

A conventional PAT and its modified models whose width-diameter ratio $b_{2} / D_{2}$ is 0.055 are comparatively investigated. The difference between the original and modified PATs is just leaning impeller and volute or not, while other parameters remain the same. The design parameters in turbine mode are: flow rate $Q=90 \mathrm{~m}^{3} / \mathrm{h}$, head $H=35 \mathrm{~m}$, rotating speed $n=1500 \mathrm{r} / \mathrm{min}$ and specific speed $n_{s}=60$. The main geometric parameters are: impeller inlet diameter $D_{1}=102 \mathrm{~mm}$, impeller outlet diameter $D_{2}=255 \mathrm{~mm}$, impeller outlet width $b_{2}=14 \mathrm{~mm}$, inlet blade angle $\beta_{1}=19.5^{\circ}$, outlet blade angle $\beta_{2}=20^{\circ}$, blade number $z=6$, volute base circle diameter $D_{3}=266 \mathrm{~mm}$, volute outlet width $b_{3}=26 \mathrm{~mm}$ and volute outlet diameter $D_{4}=65 \mathrm{~mm}$. The first and second type of modification is carried out by leaning blade $75.5^{\circ}$ and $44^{\circ}$ respectively. The third type of modification is leaning tongue $68^{\circ}$. And the final modification is realized by jointly leaning blade $44^{\circ}$ and tongue $68^{\circ}$. The leaned impeller and volute used in modified PATs are shown in Fig. 4. The modified PATs are named M1, M2, M3 and M4, respectively.

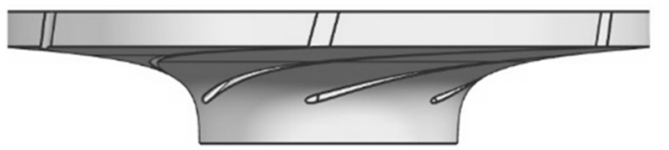

a) $75.5^{\circ}$ leaned blade

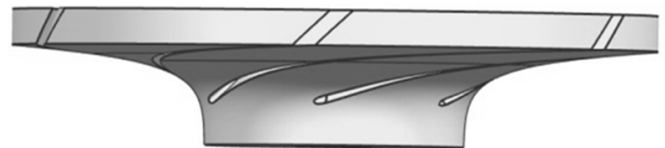

b) $44^{\circ}$ leaned blade

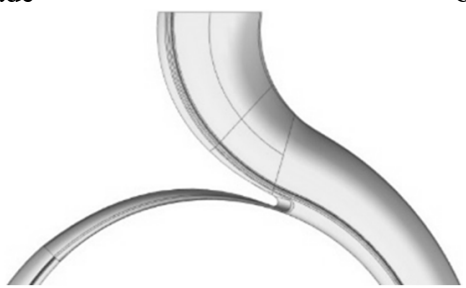

c) $68^{\circ}$ leaned tongue

Fig. 4. Leaned impeller and volute used in modified PATs

\subsection{Flow and modal calculation}

The fluid domain in whole flow passages, including the volute, impeller, front and back chambers, inlet pipe and draft tube, was modeled. Considering the complexity of geometry, unstructured tetrahedral elements were adopted to discrete the computational region. A grid independence test was performed. It was found when the element number was around $2.6 \times 10^{6}$, the variation of efficiency was within $0.5 \%$. The final mesh number of three PATs was over $2.6 \times 10^{6}$. To distinguish unsteady messages from internal flow, the time step was set to $1.1111 \times 10^{-4} \mathrm{~s}$, which means the impeller rotates about $1^{\circ}$ in each time step [17]. After the flow changes in a stable periodic mode, the temporal pressure fluctuations on internal wall surfaces using $k-\varepsilon$ model were output. Four cycles of pressure fluctuations, that is, the sampling time $T=0.16 \mathrm{~s}$, were saved for the following noise calculation.

The casing structure used for noise calculation due to structural vibration consists of pump body, cover, suspension, supporting feet, inlet pipe and draft tube. When modeling, it only retains the structures with larger normal radiation area. The other tiny structures, such as the bosses, transition fillets, were removed. Moreover, the holes in structure surface, such as holes for injection and drainage and cover surface, were filled. Solid 187 was used for modal analysis, and 
the number of elements was 1914 480. The Gray Cast HT200 was applied, whose density $\rho=7200 \mathrm{~kg} / \mathrm{m}^{3}$, elastic module $E=148 \mathrm{GPa}$ and Poisson ratio $\mu=0.3$.

\subsection{Noise calculation}

Based on wave equation, Boundary Element Method (BEM) and Finite Element Method (FEM) can accurately solve various noise problems in low and medium frequencies. However, due to various media's characteristics between internal water and external air, it is inadvisable to simultaneously solve interior/exterior noise through BEM, which can only be set one fluid material. At present, the interior noise caused by rotating dipole source of impeller can only be solved by BEM. And, it is more effective in dealing with exterior noise problems in unbounded physical domain using FEM. Therefore, BEM was used to solve interior noise, and exterior noise was calculated by FEM.

As major noise source, the dipole source can roughly be excited by rotating impeller and fixed casing. For the interior noise generated by casing source, firstly four cycles of pressure fluctuations on inner walls of casing were output. Then, the closed acoustic field can be solved with the pressure fluctuations set as acoustic boundary condition transformed by Fast Fourier Transform (FFT). As for the interior noise due to impeller's rotation, only excitation in time domain was needed to be extracted without FFT. To make compact compared with acoustic wavelength, the impeller was divided into 10 pieces. To simulate the direct propagation of noise in piping without reflection, the inlet pipe and draft tube were set to full absorption, and other surfaces were defined as total reflection, with the acoustic impedance $Z=1.5 \times 10^{6} \mathrm{~kg} /\left(\mathrm{m}^{2} \cdot \mathrm{s}\right)$. Monitoring point was set at the same position with the experiment.

For exterior noise calculation, firstly the pressure fluctuations were transferred to inner walls of casing structure. Then, the vibration response was solved based on structure modal. Finally, with the vibration displacement on inner walls as boundary condition, exterior noise was solved. During the calculation, the internal boundary of finite element mesh fitted well with the casing shape. And, the external boundary was established as convex with AML attribute specified. Fig. 5 shows the acoustic boundary conditions for noise calculation.

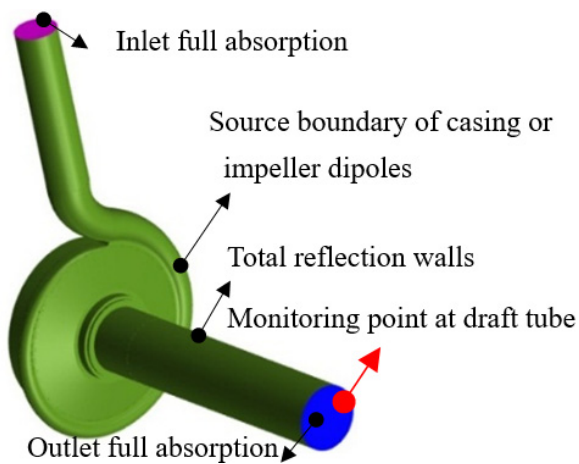

a) Interior noise

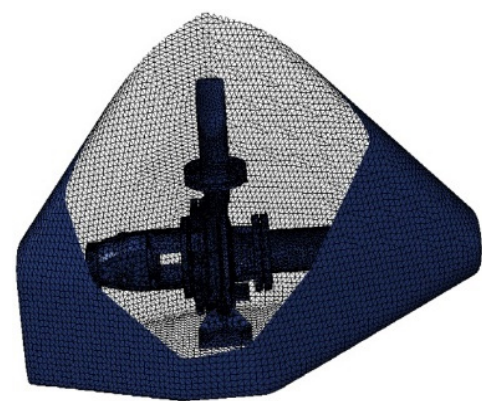

b) Exterior noise

Fig. 5. Acoustic boundary conditions for noise calculation

Due to obvious directivity of sound transmission, the measured sound pressure level (SPL) is not the same at different locations with respect to sound sources in space. To obtain the distribution of SPL, the impeller rotating center $(0,0,0)$ was regarded as the origin to establish monitoring planes of $1 \mathrm{~m} \times 1 \mathrm{~m}$ respectively paralleling to $x, y, z$. These planes were defined as horizontal, vertical, and axial direction. 36 monitoring points were disposed on each monitoring plane at $1 \mathrm{~m}$ away from the center of impeller, and the angle between each point was 10 , as shown in Fig. 6 . The four monitoring points were established to monitor the frequency response curves of exterior noise. 


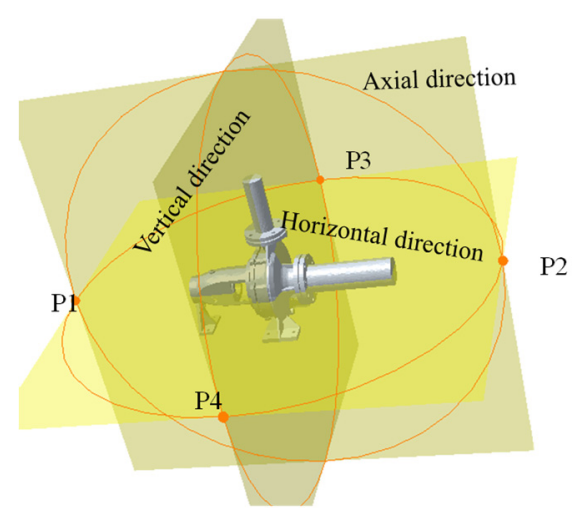

Fig. 6. Monitoring planes and points

\section{Experimental verification}

\subsection{Hydraulic performance and interior noise}

The experimental measurements of hydraulic performance and interior noise were carried out, as shown in Fig. 7. Derakhshan [18] established a correlation between the performances of direct (pump) and reverse (turbine) modes. To measure the turbine's shaft torque, the generator was modified to suspense state mode. Bozorgi [19] built a test bench for axial pump as turbine using similar method. In their testing systems, the energy recovered by PAT was measured by the energy conversion way from pressure energy to electric energy. There are two main demerits: (1) because the motor's speed cannot be maintained constant only by the load controller, and the flow and head of output load need to be adjusted, the output energy of PAT is not stable; (2) the flow only adjusted through regulating valve makes the flow not easy to control. Singh [20] added a surge tank in his test to make the head of inlet constant and adjust flow. However, the test bench is difficult to establish with complex pipelines and high cost.

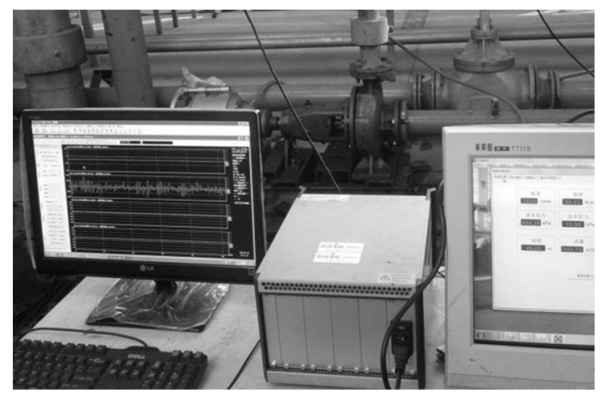

Fig. 7. Sketch of the test ring

To cure the above problems, the PAT was connected with dynamometer in the paper. A CWF22L eddy current dynamometer was used to consume and measure the energy recovered by PAT. The impeller of PAT was rotated due to the impact of high pressure water supplied from the booster pump. In the control mode of constant speed, the EST measuring and control system can make the speed of PAT remain constant over time. The discharge and pressure of inlet and outlet were respectively measured by flow meter and pressure transmitter. The uncertainty of measured parameters, such as head $H$, flow rate $Q$, hydraulic power $P_{h}$, shaft power $P$ and efficiency $\eta$ were $\pm 0.14 \%, \pm 0.50 \%, \pm 0.52 \%, \pm 1.08 \%$ and $\pm 1.20 \%$, respectively. The INV vibration and noise test system from China Orient Institute of Noise and Vibration was used to acquire noise signals. The system included INV3020C high-performance 24-position acquisition system and DASP large-capacity data processing software. A ST70 hydrophone was flush mounted on the piping, 
which was four times of outlet diameter away in turbine mode. In the process of measuring under each flow rate, the valves remained fully opened, eliminating the influence of valve noise in pipeline. The data acquisition frequency was chosen equal to $25.6 \mathrm{kHz}$ per channel with a resolution of $12.5 \mathrm{~Hz}$. To reduce random noise error, the spectrums of four divided pieces of data were averaged using the whole average method. Besides that, to reduce leakage error in frequency-domain due to time domain truncation, Hanning window was adopted to process data.

The conventional PAT was selected for validation of calculation accuracy. Fig. 8 shows a comparison of hydraulic performance curves between calculation and experiment. We can see, the tendency of calculated curves is in agreement with that of experiment, especially in larger flow rate. The numerical efficiency, head, and shaft power are lower for part-load conditions than experimental results, with a largest difference of $8.44 \%$ at $Q=70 \mathrm{~m}^{3} / \mathrm{h}$. The comparison indicates the mesh and turbulence model are suitable for hydraulic performance prediction.

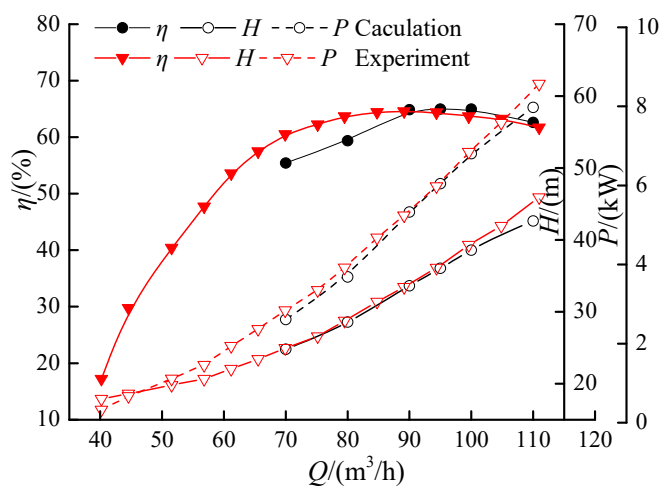

Fig. 8. Comparison of hydraulic performance between calculation and experiment

Fig. 9 shows a comparison of spectrum curves of interior noise between calculation and experiment under three flow conditions. It is found that the calculated spectrum curves obtained from both impeller and casing dipole sources are in good agreement with experimental results, especially in optimal and large flow condition. In the range of $12.5 \mathrm{~Hz}$ to $450 \mathrm{~Hz}$, the SPL at a given frequency obtained from experiment is higher than calculated value on the whole. The calculated SPL at dominant frequency is basically identical with experimental value, while larger differences exist among low frequencies. Within the range of $450 \mathrm{~Hz}$ to $2000 \mathrm{~Hz}$, the calculated broadband spectrum agrees well with the experiment, while larger SPL deviation exists at Blade-Passing Frequency (BPF) and its harmonics.

According to sound energy superposition principle, the noise under the action of two sound sources is determined by the sound source with higher SPL. At shaft frequency, the final noise is determined by impeller source, with minimum error of $12.6 \%$. At BPF and its harmonics, the final noise is basically determined by casing source, with relatively smaller errors in optimal and large flow conditions. During these flow conditions, the biggest error is $9.3 \%$, the minimum error is $-0.9 \%$, and the average error is only $4.35 \%$. It can be inferred that, the interior noise due to casing source can reflect joint action of multiple sources. And, it is feasible to predict interior noise induced only by casing source through BEM. The causes of error are analyzed as below:

(1) In the process of measurement, the impeller is unlikely completely dynamically balanced. Certain levels of vibration will be generated at the hydrophone installed on draft tube, lowering measurement accuracy. (2) The actual noise excited by unsteady flow will radiate inside after repeated reflection and scatter on irregular walls. The scattering effect is ignored during noise calculation. So, the interior noise may be enhanced at some frequencies, and suppressed at others. (3) The internal flow of PAT is complex at smaller flow rates, including flow separation, backflow, and so on. Only the hydrodynamic noise based on URANS was calculated, so the calculated value may be less. 


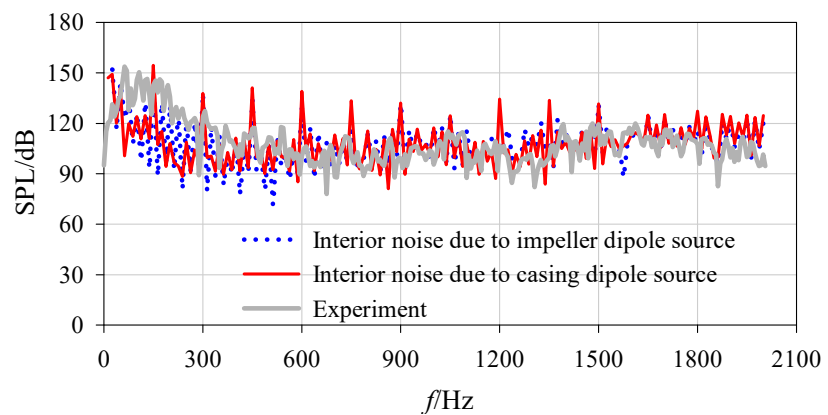

a) $80 \mathrm{~m}^{3} / \mathrm{h}$

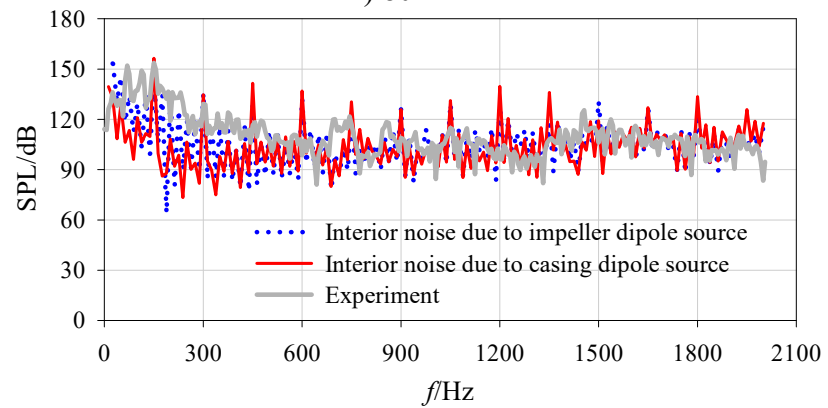

b) $90 \mathrm{~m}^{3} / \mathrm{h}$

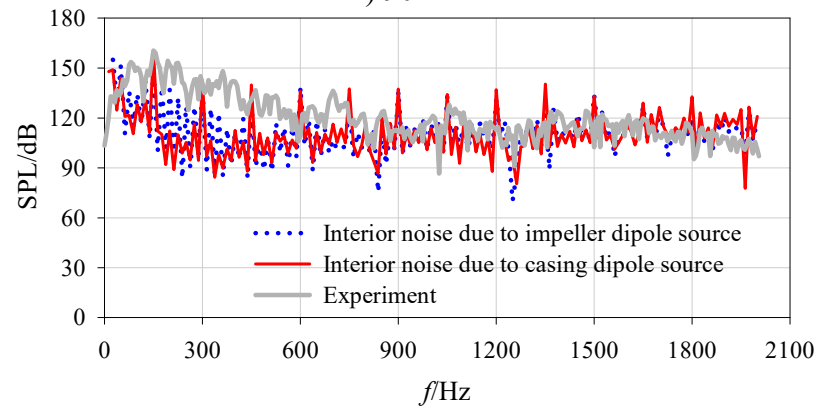

c) $110 \mathrm{~m}^{3} / \mathrm{h}$

Fig. 9. Comparison of spectrum curves between calculation and experiment

\subsection{Structure modal used for exterior noise}

The modal test of casing was performed by moving incentive points with a single response point fixed. The acceleration transducer and force-hammer were connected to signal modulation system and SD380 dynamic analysis system. Then the matrix of frequency response function (FRF) for free system was constructed. At last, the modal parameters were extracted from the FRF matrix by polynomial curve fitting techniques in frequency domain. The acquisition frequency was chosen equal to $5.12 \mathrm{kHz}$ with a resolution of $6.4 \mathrm{~Hz}$. The transient force window was imposed to improve the signal-to-noise ratio of excitation signal. And index window was imposed on response signal to accelerate vibration attenuation and avoid leakage of frequency response function. Table 1 gives the first seven modal frequencies in free condition. And, Fig. 10 shows three representative modes of vibration. As can be seen, the modes of vibration are approximate between calculation and experiment. The calculated errors of modal frequencies are within $7 \%$, meaning the finite element model is available for noise analysis based on modal.

In practice, the PAT is fixed on ground by bolts, and the inlet and outlet are connected with inlet pipe and draft tube. So the boundary conditions of modal analysis were set as: the bottom contacted with ground was supposed six constraints, the up and down displacement was restricted 
on inlet pipe, and the axial displacement was restricted on draft tube. The constraint natural frequencies are $311.10 \mathrm{~Hz}, 416.83 \mathrm{~Hz}, 762.87 \mathrm{~Hz}, 886.73 \mathrm{~Hz}, 1363.75 \mathrm{~Hz}, 1603.67 \mathrm{~Hz}$ and 1715.77 Hz, respectively.

Table 1. Comparison of free modal frequency between calculation and experiment

\begin{tabular}{|c|c|c|c|c|c|}
\hline \multirow{2}{*}{ Model } & \multirow{2}{*}{ Order } & \multicolumn{2}{|c|}{ Frequency / Hz } & \multirow{2}{*}{ Error / \% } & \multirow{2}{*}{ Damping ratio / \% } \\
\cline { 3 - 6 } & & Experiment & Calculation & & \\
\hline \multirow{5}{*}{ Casing } & 1 & 285.62 & 265.84 & -6.93 & 1.62 \\
\cline { 2 - 6 } & 2 & 618.65 & 638.84 & 3.26 & 0.70 \\
\cline { 2 - 6 } & 3 & 864.23 & 875.59 & 1.31 & 0.30 \\
\cline { 2 - 6 } & 4 & 1010.0 & 946.66 & -6.27 & 0.68 \\
\cline { 2 - 6 } & 5 & 1050.0 & 1073.9 & 2.28 & 0.28 \\
\cline { 2 - 6 } & 6 & 1110.0 & 1194 & 7.57 & 0.26 \\
\hline
\end{tabular}

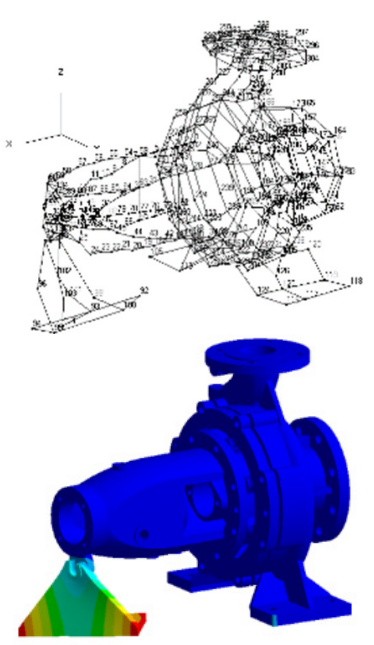

a) Second-order

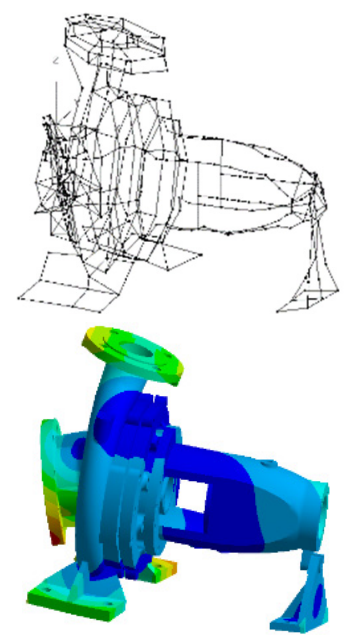

b) Third-order

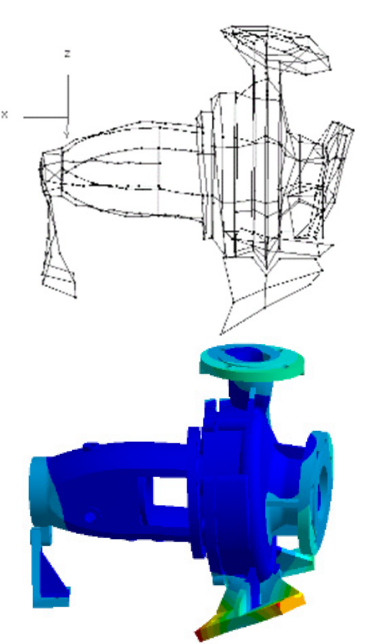

c) Sixth-order

Fig. 10. Comparison of free mode of vibration between calculation and experiment

\section{Results and discussions}

\subsection{Influence of leaning blade or tongue on hydraulic performance}

The hydraulic performance curves of PATs were obtained by numerical calculation, as shown in Fig. 11. Table 2 lists the corresponding Best Efficiency Points (BEP). From Fig. 11, after leaning blades, the efficiency of the PAT at most flow rates declines slightly with a biggest drop of $0.6 \%$. Whereas at larger flow rates $\left(Q>90 \mathrm{~m}^{3} / \mathrm{h}\right)$, the efficiency increases, reaching a maximum increase of $0.15 \%$ observed at the largest flow rate $Q=110 \mathrm{~m}^{3} / \mathrm{h}$. In addition, along with enlarging blade's leaning angle, its amplitude of increase improves at larger flow rates. Compared with conventional PAT, the efficiency of the PAT with a leaned tongue increases over full flow rates. And, the increase is from $0.67 \%$ to $1.81 \%$ with a relative smaller increase after BEP than before. After counter-leaning blade and tongue, the efficiency is basically same with conventional PAT. But there is a certain increase of efficiency at larger flow rates. Table 3 shows that as the angle is leaned by $75.5^{\circ}$ and $44^{\circ}$, the flow rate at BEP is increased by $10 \mathrm{~m}^{3} / \mathrm{h}$ and $5 \mathrm{~m}^{3} / \mathrm{h}$, respectively. This indicates that there is a rise of flow rate at BEP with the inclination of blade. Additionally, we can see there is a rise of flow rate at BEP for leaned tongue or combined with leaned blade. All these indicate that the leaned blade can keep PAT's original performance, the leaned tongue can significantly improve PAT's efficiency over an overall flow range, and the combined leaned blade and volute can increase the efficiency in larger flow rates. 


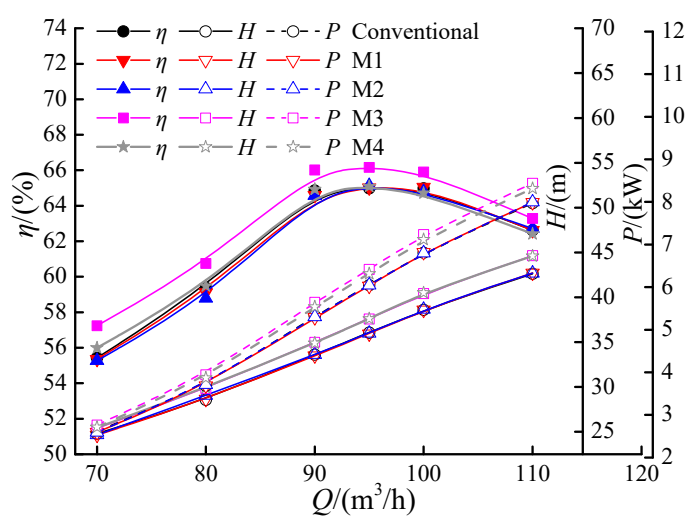

Fig. 11. Comparison of hydraulic performance curves

Table 2. PAT's BEPs with different blade and tongue

\begin{tabular}{|c|c|c|c|c|}
\hline Models & $Q\left(\mathrm{~m}^{3} / \mathrm{h}\right)$ & $H(\mathrm{~m})$ & $P_{h}(\mathrm{~kW})$ & $\eta(\%)$ \\
\hline Conventional & 90.00 & 38.59 & 6.81 & 64.98 \\
\hline M1 & 100.00 & 38.59 & 6.82 & 65.05 \\
\hline M2 & 95.00 & 35.98 & 6.05 & 65.13 \\
\hline M3 & 95.00 & 37.58 & 6.42 & 66.16 \\
\hline M4 & 95.00 & 37.61 & 6.32 & 65.09 \\
\hline
\end{tabular}

\subsection{Influence of leaning blade or tongue on interior/exterior noise}

Fig. 12 shows the comparison of SPLs at discrete frequencies in interior noise for $Q=90 \mathrm{~m}^{3} / \mathrm{h}$. When the blade and tongue are leaned, the SPLs at BPF and its second-order and fifth-order frequencies increase firstly and then decrease. At these frequencies, the SPLs under joint action of leaned blade and tongue are reduced by about $2.48 \mathrm{~dB}, 4.08 \mathrm{~dB}$ and $2.57 \mathrm{~dB}$. Whereas, by leaning blade $44^{\circ}$, the SPLs increase $0.39 \mathrm{~dB}, 1.69 \mathrm{~dB}$ and $0.4 \mathrm{~dB}$. Of course, at some other frequencies, the SPLs are reduced by leaning blade. For example, there is a noise reduction of $1.76 \mathrm{~dB}$ at $450 \mathrm{~Hz}$.

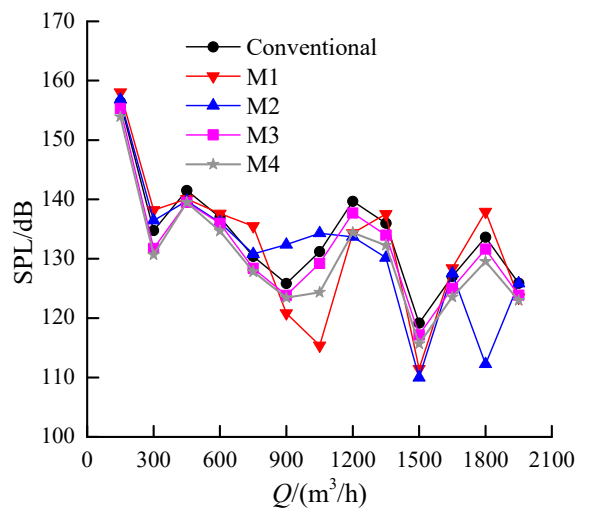

Fig. 12. Comparison of SPLs in interior noise, $Q=90 \mathrm{~m}^{3} / \mathrm{h}$

Fig. 13 shows the comparison of spectrum curves in exterior noise for conventional PAT at four monitoring points generated by casing dipole for $Q=90 \mathrm{~m}^{3} / \mathrm{h}$. The peak value occurs at fifth-order BPF followed by second order BPF, mainly because they are close to the third and first constraint natural frequency of casing. It indicates the exterior noise is determined both by internal fluid and solid structure.

Due to approximate directivity on three monitoring planes for exterior noise, the horizontal 
directivity was analyzed here focusing on fifth-order BPF. Fig. 14 gives the sound pressure distribution under three typical working conditions. At the fifth-order BPF, the space directivity of modified PATs is nearly the same compared with conventional PAT, showing a side lobe phenomenon. At all flow rates, the exterior noise decreases for leaned blade $44^{\circ}$, while increases for leaned blade $75.5^{\circ}$. Respectively for leaned blade $44^{\circ}$ and $75.5^{\circ}$, the noise reduces by $2.84 \mathrm{~dB}$ and increases by $0.76 \mathrm{~dB}$ at $Q=80 \mathrm{~m}^{3} / \mathrm{h}$, and $2.97 \mathrm{~dB}$ and $0.91 \mathrm{~dB}$ at $Q=110 \mathrm{~m}^{3} / \mathrm{h}$. At $Q=80 \mathrm{~m}^{3} / \mathrm{h}$, the noise is reduced by $5.00 \mathrm{~dB}$ and $5.12 \mathrm{~dB}$, respectively for leaned tongue or joint leaned blade and tongue. At $Q=90 \mathrm{~m}^{3} / \mathrm{h}$ and $Q=110 \mathrm{~m}^{3} / \mathrm{h}$, the noise reduction effect is better for joint action. The noise reduction is approximately $3.68 \mathrm{~dB}$ and $5.76 \mathrm{~dB}$ for the two flow rates.

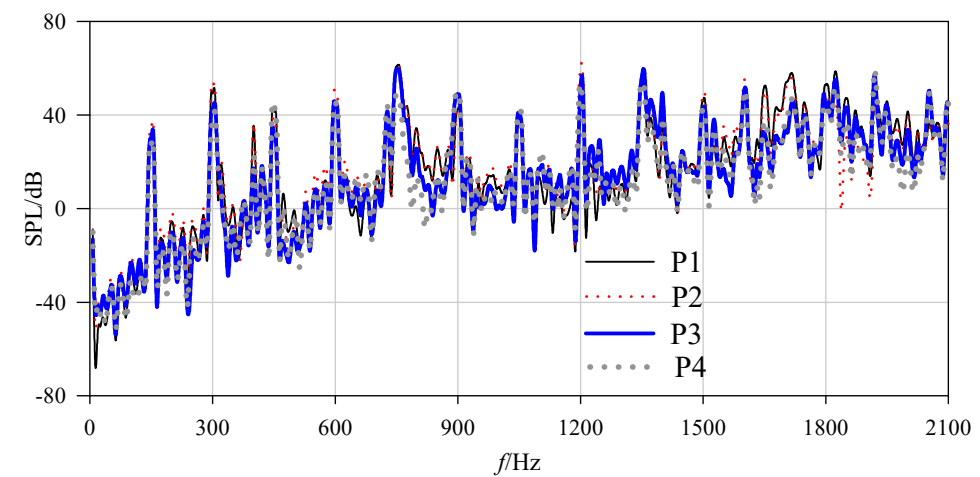

Fig. 13. Comparison of SPLs in exterior noise for conventional PAT, $Q=90 \mathrm{~m}^{3} / \mathrm{h}$

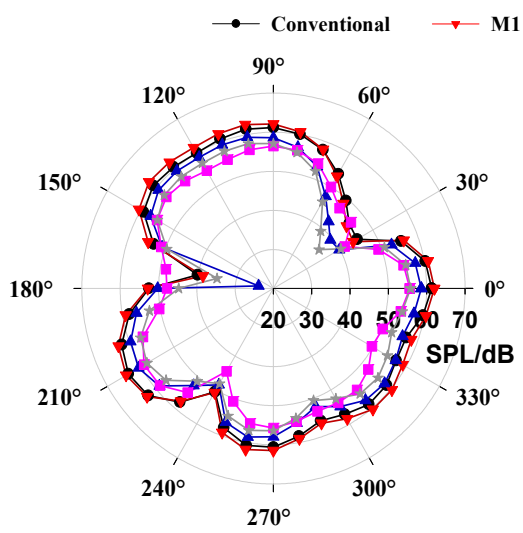

a) $80 \mathrm{~m}^{3} / \mathrm{h}$

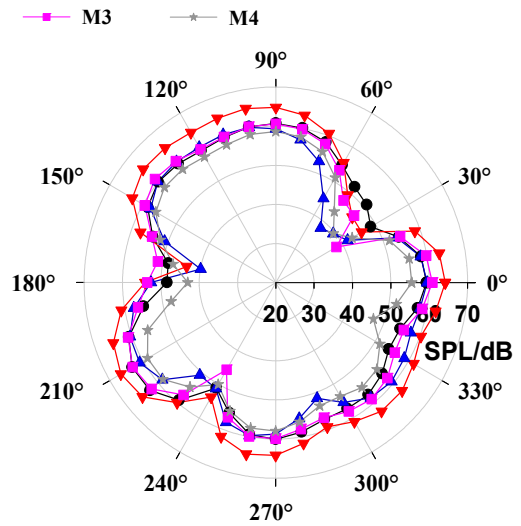

b) $90 \mathrm{~m}^{3} / \mathrm{h}$

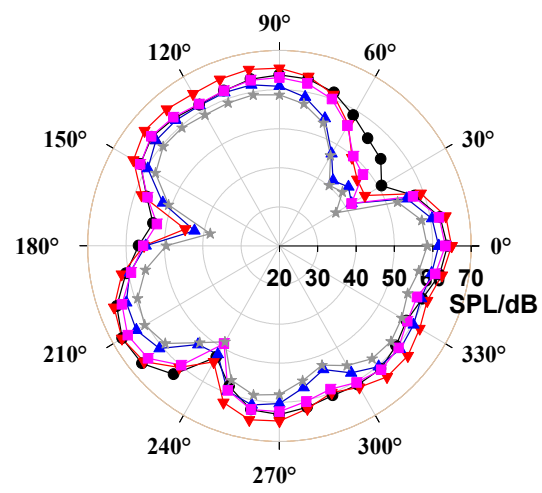

c) $110 \mathrm{~m}^{3} / \mathrm{h}$

Fig. 14. Comparison of directivity distribution at fifth-order BPF in exterior noise 
The overall interior/exterior noise characteristics were compared, as shown in Table 3 . The interior noise was evaluated by overall sound pressure level. The exterior noise was estimated by overall sound power level. Sound power level is used to characterize the amount of radiation energy from sound source, independent of propagation distance of sound wave and environment. On the basis of reference pressure in air, the A-weighted was applied within the frequency range from $12.5 \mathrm{~Hz}$ to $2000 \mathrm{~Hz}$, because of its closer feeling to human's ears. At the same flow rate, the interior/exterior noise of PATs with leaned blade or tongue reduces greatly, and the decreasing amplitude under the combined effect of leaned blade and tongue is basically greatest. The overall sound pressure level under the combined effect reduces $7.19 \%$, and the overall sound power level decreases $12.15 \%$ at the largest flow rate $Q=110 \mathrm{~m}^{3} / \mathrm{h}$. The noise reduction effect is nearly the same for only leaning blade $44^{\circ}$ or leaning tongue $68^{\circ}$ at all flow rates. The highest decreasing amplitude can be observed at the largest flow rate $Q=110 \mathrm{~m}^{3} / \mathrm{h}$ for all modified PATs, and the smallest decreasing amplitude has been obtained for $Q=80 \mathrm{~m}^{3} / \mathrm{h}$. As can be seen, the peak value at fundamental frequency can be reduced by leaned blade or tongue. In addition, the leaned blade or tongue can reduce the total sound energy in full-frequency band. Considering both the hydraulic and noise performance, the comprehensive performance of PAT under the combination of leaned blade and tongue is optimal.

Table 3. Interior/exterior noise characteristics of PATs with different blade and tongue

\begin{tabular}{|c|c|c|c|}
\hline$Q\left(\mathrm{~m}^{3} / \mathrm{h}\right)$ & Models & Overall sound pressure level (dBA) & Overall sound power level (dBA) \\
\hline \multirow{5}{*}{80} & Conventional & 146.74 & 67.64 \\
\hline & M1 & 142.90 & 67.55 \\
\hline & M2 & 142.31 & 66.75 \\
\hline & M3 & 141.07 & 67.08 \\
\hline & M4 & 140.21 & 66.91 \\
\hline \multirow{5}{*}{90} & Conventional & 152.50 & 72.46 \\
\hline & M1 & 146.28 & 68.86 \\
\hline & M2 & 144.61 & 67.75 \\
\hline & M3 & 143.46 & 68.38 \\
\hline & M4 & 142.95 & 65.97 \\
\hline \multirow{5}{*}{110} & Conventional & 154.57 & 77.18 \\
\hline & M1 & 147.25 & 69.57 \\
\hline & M2 & 145.90 & 67.94 \\
\hline & M3 & 145.55 & 68.43 \\
\hline & M4 & 143.46 & 67.80 \\
\hline
\end{tabular}

\subsection{Influence of leaning blade or tongue on hydraulic exciting force}

The hydraulic exciting force acting on structure surface is the direct reason causing interior noise. Fig. 15 shows the vector diagrams of radial exciting force on impeller in rotating coordinate system.

In terms of the distribution of radial force, it still shows a hexagonal distribution after leaning blade or tongue. In terms of the value of radial force, it decreases to some extent. At $Q=80 \mathrm{~m}^{3} / \mathrm{h}$, the leaned tongue only makes the radial force reduce by $0.69 \%$ compared with conventional PAT. As the blade is leaned from $90^{\circ}$ to $44^{\circ}$, the radial force decreases gradually with bigger reduction. The leaned blade with $44^{\circ}$ makes the radial force reduce by $17.94 \%$ compared with conventional PAT. Under the joint action of leaned blade $44^{\circ}$ and leaned tongue $68^{\circ}$, the radial force is reduced by $21.53 \%$. As the flow rate increases, the reduction effect is more obvious by leaning blade or tongue, and the reduction is up to $40.70 \%$ under the combined action. 


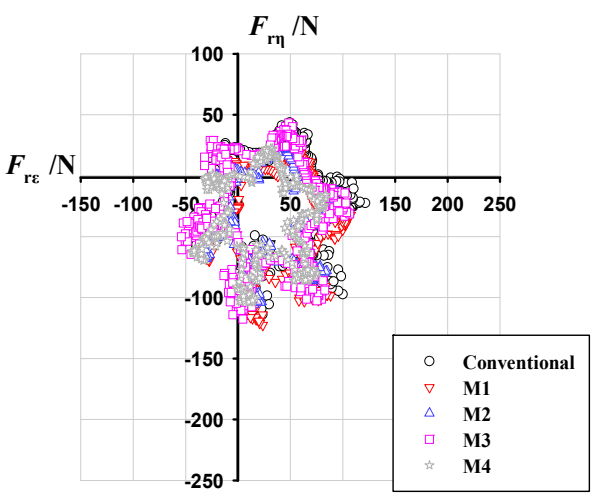

a) $80 \mathrm{~m}^{3} / \mathrm{h}$

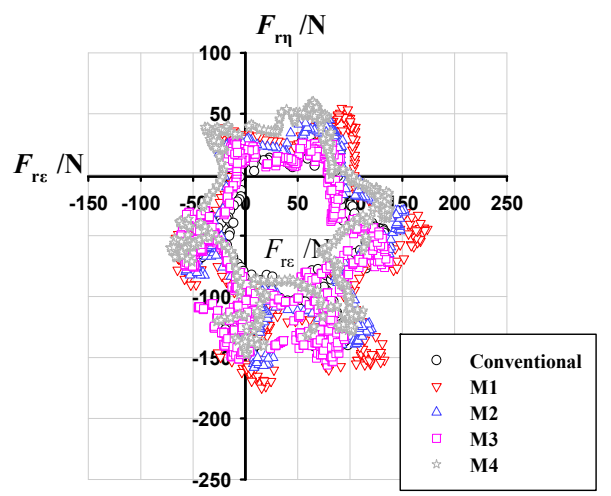

b) $90 \mathrm{~m}^{3} / \mathrm{h}$

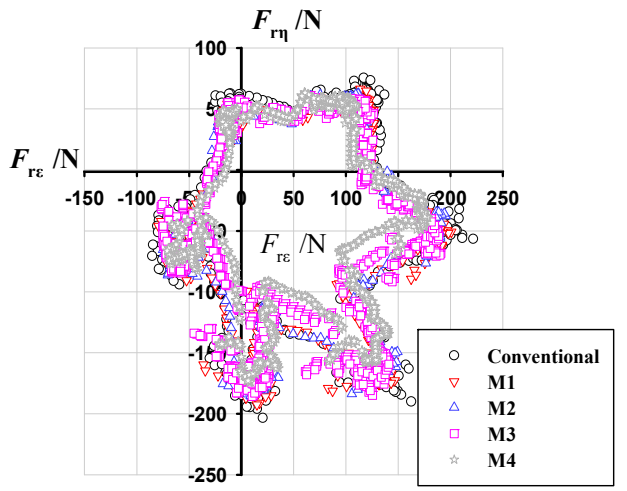

c) $110 \mathrm{~m}^{3} / \mathrm{h}$

Fig. 15. Comparison of hydraulic exciting force

\section{Conclusions}

The active noise reduction approaches were put forward by leaning blade or tongue, and it can reduce interior/exterior noise without lowering hydraulic performance. The conclusions are as follows:

1) The calculated hydraulic performance curve of conventional PAT coincides well with the experiment, especially at larger flow rate. At BPF and its harmonics, the interior noise is basically determined by casing source with relatively smaller errors at optimal and larger flow rates. The interior noise due to casing source can reflect joint action of multiple sources. The modes of vibration are approximate between calculation and experiment, indicating the finite element model of casing structure is suitable for exterior noise analysis based on modal.

2) The efficiency of the PATs by leaning blades declines at most flow rates, with a slight increases at larger flow rates. After a certain inclination of tongue, the efficiency increases over full flow rates. After counter-leaning blade and tongue, the efficiency remains basically the same with conventional PAT. The SPL at each discrete frequency in interior/exterior noise and radial exciting force acting on structure surface can be reduced by simply leaning blade or tongue and joint leaning blade and tongue. And they can reduce the total sound energy in full-frequency band. Considering both hydraulic and noise performance, the comprehensive performance of PAT under the joint action is optimal.

\section{Acknowledgements}

This work was supported by National Natural Science Foundation of China (Nos. 51509111, 51309119), China Postdoctoral Science Foundation (2015M581734), the Advanced Talent 
Foundation of Jiangsu University (12JDG082, 15JDG052), the Open Research Subject of Key Laboratory of Fluid and Power Machinery, Ministry of Education, Xihua University (szjj2015-017), Sichuan Provincial Key Lab of Process Equipment and Control (GK201403), Major Science and Technology Projects of Zhejiang Province (2014C01004-1) and a Project funded by the Priority Academic Program Development of Jiangsu Higher Education Institutions (PAPD).

\section{References}

[1] Nautiyal H., Anoop Kumar V. Reverse running pumps analytical, experimental and computational study: a review. Renewable and Sustainable Energy Reviews, Vol. 14, Issue 7, 2010, p. 2059-2067.

[2] Lu F. A. Numerical and Experimental Study on the Flow-Induced Vibroacoustics of a Centrifugal Fan and Compressor. Xi' an Jiaotong University, Xi'an, 2014.

[3] Tourret J., Badie-Cassagnet A., Bernard G., et al. Experimental studies of noise emission and noise generation from a centrifugal pump. ASME Paper No. 95-WA/FE-8, 1985.

[4] Dong R., Chu S., Katz J. Effect of modification to tongue and impeller geometry on unsteady flow, pressure fluctuations, and noise in a centrifugal pump. Journal of Turbomachinery, Vol. 119, Issue 3, 1997, p. 506-515.

[5] Langthjem M. A., Olhoff N. A numerical study of flow-induced noise in a two-dimensional centrifugal pump. Part I. Hydrodynamics. Journal of Fluids and Structures, Vol. 19, Issue 3, 2004, p. 349-368.

[6] Langthjem M. A., Olhoff N. A numerical study of flow-induced noise in a two-dimensional centrifugal pump. Part II. Hydroacoustics. Journal of Fluids and Structures, Vol. 19, Issue 3, 2004, p. 369-386.

[7] Neise W. Noise reduction in centrifugal fans: a literature survey. Journal of Sound and Vibration, Vol. 45, Issue 3, 1976, p. 375-403.

[8] Yang S. S., Kong F. Y., Shao F., et al. Numerical simulation and comparison of pump and pump as turbine. ASME 2010 3rd Joint US-European Fluids Engineering Summer Meeting collocated with 8th International Conference on Nanochannels, Microchannels, and Minichannels, Montreal, Quebec, Canada, 2010.

[9] Derakhshan S., Nourbakhsh A. Theoretical, numerical and experimental investigation of centrifugal pumps in reverse operation. Experimental Thermal and Fluid Science, Vol. 32, Issue 8, 2008, p. 1620-1627.

[10] Singh P., Nestmann F. An optimization routine on a prediction and selection model for the turbine operation of centrifugal pumps. Experimental Thermal and Fluid Science, Vol. 34, Issue 2, 2010, p. 152-164.

[11] Yang S. S., Kong F. Y., Chen H., et al. Effects of blade wrap angle influencing a pump as turbine. Journal of Fluids Engineering, Vol. 134, Issue 6, 2012, p. 061102.

[12] Yang S. S., Kong F. Y., Jiang W. M., et al. Effects of impeller trimming influencing pump as turbine. Computers and Fluids, Vol. 67, 2012, p. 72-78.

[13] Yang S. S., Kong F. Y., Fu J. H., et al. Numerical research on effects of splitter blades to the influence of pump as turbine. International Journal of Rotating Machinery, 2012, p. 123093.

[14] Dai C., Kong F. Y., Dong L., et al. Effect of blade wrap angle on the radial force of centrifugal pump as turbine. Journal of Vibration and Shock, Vol. 34, Issue 18, 2015, p. 69-99.

[15] Dai C., Kong F. Y., Dong L., et al. Hydraulic and acoustic property optimization for centrifugal pump as turbine based on response surface method. Transactions of the Chinese Society of Agricultural Engineering, Vol. 31, Issue 15, 2015, p. 40-47.

[16] Dong L., Dai C., Kong F. Y., et al. Impact of blade outlet angle on acoustic of centrifugal pump as turbine. Transactions of the Chinese Society of Agricultural Engineering, Vol. 31, Issue 6, 2015, p. 69-75.

[17] Dai C., Kong F. Y., Dong L. Pressure fluctuation and its influencing factor analysis in circulating water pump. Journal of Central South University, Vol. 20, Issue 1, 2013, p. 149-155.

[18] Derakhshan S., Nourbakhsh A. Experimental study of characteristic curves of centrifugal pumps working as turbines in different specific speeds. Experimental Thermal and Fluid Science, Vol. 32, Issue 3, 2008, p. 800-807.

[19] Bozorgi A., Javidpour E., Riasi A., et al. Numerical and experimental study of using axial pump as turbine in Pico hydropower plants. Renewable Energy, Vol. 53, 2013, p. 258-264. 
[20] Singh P. Optimization of the Internal Hydraulic and of System Design in Pumps as Turbines with Field Implementation and Evaluation. University of Karlsruhe, Karlsruhe, 2005.
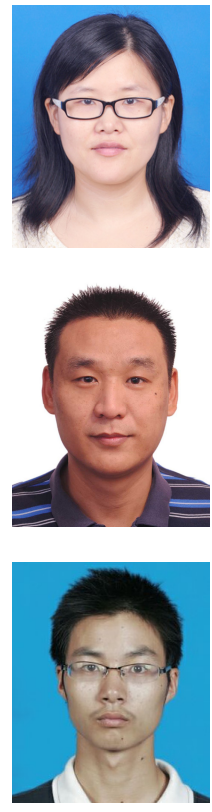

Liang Dong received Ph.D. degree in Research Center of Fluid Machinery Engineering and Technology from Jiangsu University, Zhenjiang, China, in 2012. Now he works at Research Center of Fluid Machinery Engineering and Technology. His current research interests include noise prediction and control in hydraulic machinery, numerical method and grid generation technique of pump.

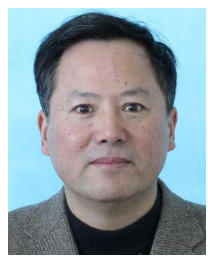

Fan-yu Kong (Professor, Doctoral supervisor) received Bachelor's degree majored in hydraulic machinery from Huazhong University of Science and Technology, Wuhan, China, in 1982. Now he works at Research Center of Fluid Machinery Engineering and Technology, Jiangsu University. His current research interests include research and

development of special pumps in military and petrifaction industry.

Bin Xia is now purchasing his Doctor's degree in Research Center of Fluid Machinery Engineering and Technology from Jiangsu University, Zhenjiang, China. His current research interests include internal unsteady flow, and low-noise structure design in pumps. 